PROCEEDINGS OF THE

AMERICAN MATHEMATICAL SOCIETY

Volume 128, Number 5, Pages 1333-1338

S 0002-9939(99)05181-3

Article electronically published on October 6, 1999

\title{
THE SIXTH, EIGHTH, NINTH, AND TENTH POWERS OF RAMANUJAN'S THETA FUNCTION
}

\author{
SCOTT AHLGREN
}

(Communicated by David E. Rohrlich)

\begin{abstract}
In his Lost Notebook, Ramanujan claimed that the "circular" summation of the $n$-th powers of the symmetric theta function $f(a, b)$ satisfies a factorization of the form $f(a, b) F_{n}(a b)$. Moreover, Ramanujan recorded identities expressing $F_{2}(q), F_{3}(q), F_{4}(q), F_{5}(q)$, and $F_{7}(q)$ in terms of his theta functions $\varphi(q), \psi(q)$, and $f(-q)$. Ramanujan's claims were proved by Rangachari, and later (via elementary methods) by Son. In this paper we obtain similar identities for $F_{6}(q), F_{8}(q), F_{9}(q)$, and $F_{10}(q)$.
\end{abstract}

\section{INTRODUCTION}

Let Ramanujan's general theta function be given by

$$
f(a, b):=\sum_{k=-\infty}^{\infty} a^{\frac{k(k+1)}{2}} b^{\frac{k(k-1)}{2}} .
$$

In the Lost Notebook of Ramanujan [Ram, p. 54], the following is recorded (without proof).

Ramanujan's claim. If $n$ is a positive integer and $|a b|<1$, then

$$
\sum_{r=0}^{n-1}\left(\sum_{\substack{k=-\infty \\ k \equiv r(\bmod n)}}^{\infty} a^{\frac{1}{n} \cdot \frac{k(k+1)}{2}} b^{\frac{1}{n} \cdot \frac{k(k-1)}{2}}\right)^{n}=f(a, b) F_{n}(a b),
$$

where $F_{n}(q)=1+2 n q^{\frac{n-1}{2}}+\ldots$ for $n \geq 3$.

This claim was proved by Rangachari $[$ Ran]. More recently, an elementary proof has been given by Son $\underline{\underline{S}}$.

Received by the editors July 10, 1998.

1991 Mathematics Subject Classification. Primary 11B65, 33D10.

Key words and phrases. Ramanujan, theta functions.

(C)2000 American Mathematical Society 
We recall the definitions of the three theta functions of Ramanujan:

$$
\begin{gathered}
\varphi(q):=f(q, q)=\sum_{k=-\infty}^{\infty} q^{k^{2}}, \\
\psi(q):=\frac{1}{2} f(1, q)=\sum_{k=0}^{\infty} q^{\frac{k(k+1)}{2}}, \\
f(-q):=f\left(-q,-q^{2}\right)=\sum_{k=-\infty}^{\infty}(-1)^{k} q^{\frac{k(3 k-1)}{2} .}
\end{gathered}
$$

With this notation, Ramanujan gave the following formulae (again without proof).

\section{Ramanujan's identities.}

$$
\begin{aligned}
& F_{2}(q)=\varphi(\sqrt{q}), \\
& F_{3}(q)=\frac{\psi^{3}(q)}{\psi\left(q^{3}\right)}+3 q \frac{\psi^{3}\left(q^{3}\right)}{\psi(q)}, \\
& F_{4}(q)=\varphi^{3}\left(q^{2}\right)+(2 \sqrt{q})^{3} \psi^{3}\left(q^{4}\right), \\
& F_{5}(q)=\frac{f^{5}(-q)}{f\left(-q^{5}\right)}+5 q \frac{f^{5}\left(-q^{5}\right)}{f(-q)}, \\
& F_{7}(q)=\frac{f^{7}(-q)}{f\left(-q^{7}\right)}+7 q^{2} \frac{f^{7}\left(-q^{7}\right)}{f(-q)}+7 q f^{3}(-q) f^{3}\left(-q^{7}\right) .
\end{aligned}
$$

These identities were proved by Rangachari Ran using methods from coding theory. Son [S] has recently succeeded in finding elementary proofs. In a recent paper, Ono $\left[\right.$ has obtained a simple expression for $F_{11}$.

In this paper we shall exhibit and prove identities which express $F_{6}, F_{8}$, and $F_{9}$ in terms of $\varphi, \psi$, and $f$. We also obtain such an expression for $F_{10}$, but it is not as compact as the rest, and we shall reserve its display until the last section (see Theorem 2 below).

Theorem 1. Let the notation be as above. Then we have

$$
32 F_{6}(q)=-3 \varphi^{5}(\sqrt{q})+5 \varphi^{3}(\sqrt{q}) \varphi^{2}\left(\sqrt{q^{3}}\right)+15 \varphi(\sqrt{q}) \varphi^{4}\left(\sqrt{q^{3}}\right)+15 \frac{\varphi^{6}\left(\sqrt{q^{3}}\right)}{\varphi(\sqrt{q})}
$$

$$
\begin{gathered}
8 F_{8}(q)=\varphi^{7}\left(q^{2}\right)+\left[2 \sqrt{q} \psi\left(q^{4}\right)\right]^{7}+14 \varphi^{5}\left(q^{2}\right) \varphi^{2}(q)-7 \varphi^{3}\left(q^{2}\right) \varphi^{4}(q), \\
F_{9}(q)=\varphi^{8}\left(\sqrt{q^{9}}\right)-16 \sqrt{q^{9}} \psi^{8}\left(\sqrt{q^{9}}\right)+256 q^{9} \psi^{8}\left(q^{9}\right)+18 q^{4} \frac{f^{12}\left(-q^{9}\right)}{f^{4}\left(-q^{3}\right)} .
\end{gathered}
$$

In order to prove these identities, we shall show that they are equivalent to identities involving the Fourier expansions of certain modular forms; these new identities may be verified by comparing enough terms to exceed the dimension of the relevant space of forms. The main difficulty, therefore, lies not in proving the identities, but rather in discovering them. 


\section{Preliminaries}

We begin with some basics regarding modular forms. If $k \in \mathbb{Z}, N$ is a positive integer, and $\chi$ is a Dirichlet character modulo $N$, then, as usual, we denote by $M_{k}\left(\Gamma_{0}(N), \chi\right)$ the space of weight $k$ modular forms with character $\chi$ on the congruence subgroup $\Gamma_{0}(N)$ (see, for example, $[\mathrm{K}$ §3.3] for full definitions).

We shall also require half-integral weight modular forms. Suppose that $k$ is an integer (even or odd), that $\gamma=\left(\begin{array}{ll}a & b \\ c & d\end{array}\right) \in \Gamma_{0}(4)$, and that $z$ is a variable of the upper half-plane $\mathbb{H}$ of complex numbers. Then we define the automorphy factor $j(\gamma, z)$ by

$$
j(\gamma, z):=\left(\frac{c}{d}\right) \epsilon_{d}^{-1} \sqrt{c z+d}
$$

Here $\left(\frac{c}{d}\right)$ is the usual quadratic residue symbol, $\epsilon_{d}=1$ or $i$ according as $d \equiv$ 1 or $3(\bmod 4)$, and we take the branch of the square root having argument in $(-\pi / 2, \pi / 2]$. If $N$ is a positive integer such that $4 \mid N$, then we define the group $\tilde{\Gamma}_{0}(N)$ by

$$
\tilde{\Gamma}_{0}(N):=\left\{(\gamma, j(\gamma, z)): \gamma \in \Gamma_{0}(N)\right\}
$$

If $\chi$ is a Dirichlet character modulo $N$, then we denote by $M_{k / 2}\left(\tilde{\Gamma}_{0}(N), \chi\right)$ the space of functions $f(z)$ which are holomorphic on $\mathbb{H}$, and which satisfy:

(1) $f(\gamma z)=\chi(d) j(\gamma, z)^{k} f(z)$ for all $z \in \mathbb{H}, \gamma=\left(\begin{array}{ll}a & b \\ c & d\end{array}\right) \in \Gamma_{0}(N)$.

(2) $f$ is holomorphic at the cusps (see $[\mathrm{K}, \S 4.1]$ for a precise definition).

If $4 \mid N$, then we shall denote by $\chi_{-1}$ the character modulo $N$ defined by $\chi_{-1}(n)=$ $(-1)^{\frac{n-1}{2}}$ for $n \in(\mathbb{Z} / N \mathbb{Z})^{*}$ (the value of $N$ will always be clear from context). With this notation, we have the following relationship between spaces of integral and half-integral weight forms: If $k$ is even and $4 \mid N$, then

$$
M_{k / 2}\left(\tilde{\Gamma}_{0}(N), \chi\right)=M_{k / 2}\left(\Gamma_{0}(N), \chi \chi_{-1}^{k / 2}\right) .
$$

In what follows, we shall always make the identification $q=e^{2 \pi i z}$, where $z \in \mathbb{H}$. We define the classical theta function $\theta(z)$ by

$$
\theta(z)=\sum_{k=-\infty}^{\infty} e^{2 \pi i k^{2} z}=\sum_{k=-\infty}^{\infty} q^{k^{2}}
$$

The next lemma is the main tool in the proof of our identities.

Lemma 2.1. If $n \geq 2$, then

$$
\theta(z) F_{n}\left(q^{2}\right) \in M_{n / 2}\left(\tilde{\Gamma}_{0}(4 n), \chi\right), \quad \text { where } \chi(d):=\left(\frac{4 n}{d}\right)^{n} .
$$

Proof. For $a, r$, and $n \in \mathbb{Z}$ satisfying $n / a \in \mathbb{Z}$ and $a r / n \in \mathbb{Z}$, then, following the well-known paper of Shimura [Sh, $\S 2$ ], we define the theta function

$$
\theta(z ; r, a, n):=\sum_{k \equiv r(\bmod n)} q^{k^{2} a / 2 n^{2}} .
$$


If we make the specialization $a=b=q$ in (1.2), then we find that Ramanujan's identity implies the following:

$$
\theta(z) F_{n}\left(q^{2}\right)=\sum_{r=0}^{n-1}\left(\sum_{k \equiv r(\bmod n)} q^{k^{2} / n}\right)^{n}=\sum_{r=0}^{n-1} \theta^{n}(z ; 2 r, 2 n, 2 n) .
$$

Suppose that $\gamma=\left(\begin{array}{ll}a & b \\ c & d\end{array}\right) \in \Gamma_{0}(4 n)$. Then for $0 \leq r \leq n-1$, Proposition 2.1 of Sh] shows that

$$
\theta(\gamma z ; 2 r, 2 n, 2 n)=e^{\frac{2 \pi i a b r^{2}}{n}}\left(\frac{4 n}{d}\right) j(\gamma, z) \theta(z ; 2 a r, 2 n, 2 n) .
$$

Denote the function in (2.2) by $f(z)$. Then for $\gamma$ as above, the last equation implies that

$$
f(\gamma z)=\left(\frac{4 n}{d}\right)^{n} j(\gamma, z)^{n} \sum_{r=0}^{n-1} \theta^{n}(z ; 2 a r, 2 n, 2 n)=\left(\frac{4 n}{d}\right)^{n} j(\gamma, z)^{n} f(z)
$$

(the last equality follows since $\operatorname{gcd}(a, n)=1$ ). This shows that $f(z)$ satisfies the proper transformation law to be an element of $M_{n / 2}\left(\tilde{\Gamma}_{0}(4 n), \chi\right)$.

It remains to check that $f$ is holomorphic at the cusps. To show this, it will suffice to show that for each $r$ and for all $\gamma=\left(\begin{array}{ll}a & b \\ c & d\end{array}\right) \in \mathrm{SL}_{2}(\mathbb{Z})$, the function

$$
(c z+d)^{-1 / 2} \theta(\gamma z ; 2 r, 2 n, 2 n)
$$

has a Fourier expansion in non-negative powers of $q^{\frac{1}{4 n}}$. This can be verified via a straightforward computation, adapting the arguments following (2.6) of [Sh].

\section{Proof of the identities}

After replacing $q$ by $q^{2}$ in (1.3), multiplying both sides by $\theta(z)$, and using the identity $\varphi(q)=\theta(z)$, we see that (1.3) is equivalent to

$$
32 \theta(z) F_{6}\left(q^{2}\right)=-3 \theta^{6}(z)+5 \theta^{4}(z) \theta^{2}(3 z)+15 \theta^{2}(z) \theta^{4}(3 z)+15 \theta^{6}(3 z) .
$$

If $m$ is a positive integer, then we have

$$
\theta(m z) \in M_{1 / 2}\left(\tilde{\Gamma}_{0}(4 m),\left(\frac{4 m}{\bullet}\right)\right) .
$$

Using this fact, we conclude that the right side of (3.1) lies in $M_{6 / 2}\left(\tilde{\Gamma}_{0}(12)\right)$. By Lemma 2.1 and (2.1), we see that both sides of (3.1) are elements of $M_{3}\left(\Gamma_{0}(24), \chi_{-1}\right)$. Using [C-O, Théorème 1], we find that the dimension of the last space is 12. So (3.1) can be verified by comparing 12 terms (here, and below, we use (2.2) to compute $\left.\theta(z) F_{n}\left(q^{2}\right)\right)$. This establishes (1.3).

We turn to $F_{8}$. Define the Dedekind eta-function by

$$
\eta(z):=q^{\frac{1}{24}} \prod_{n=1}^{\infty}\left(1-q^{n}\right) .
$$

Then Jacobi's triple product identity shows that

$$
q^{1 / 8} \psi(q)=\frac{\eta^{2}(2 z)}{\eta(z)}
$$


Using this fact after replacing $q$ with $q^{2}$ in (1.4) and multiplying through by $\theta(z)$, we obtain the following identity, which is equivalent to (1.4):

$$
\begin{aligned}
8 \theta(z) F_{8}\left(q^{2}\right)= & \theta(z) \theta^{7}(4 z)+2^{7} \theta(z) \frac{\eta^{14}(16 z)}{\eta^{7}(8 z)} \\
& +14 \theta(z) \theta^{5}(4 z) \theta^{2}(2 z)-7 \theta(z) \theta^{3}(4 z) \theta^{4}(2 z) .
\end{aligned}
$$

Using (3.2), we find that the first, third, and fourth terms on the right are contained in $M_{8 / 2}\left(\tilde{\Gamma}_{0}(16)\right)=M_{4}\left(\Gamma_{0}(16)\right)$. We can use the identity $\theta(z)=\eta^{5}(2 z) / \eta^{2}(z) \eta^{2}(4 z)$ in order to express the second term as an eta-quotient. A theorem of Gordon, Hughes, and Newman (see, for example, Theorem 3 of [G-H] ) may be used to prove that this eta-quotient satisfies the correct transformation formula to be an element of $M_{4}\left(\Gamma_{0}(16)\right.$ ). We may then use a theorem of Ligozat [L] (which is also recorded as Theorem 5 of $[\mathrm{G}-\mathrm{H}$ ) in order to verify that this eta-quotient is holomorphic at the cusps. After this we conclude that the second term is indeed a member of $M_{4}\left(\Gamma_{0}(16)\right)$. In view of Lemma 2.1, we see that both sides of (3.4) lie in $M_{4}\left(\Gamma_{0}(32)\right)$, which by $[\mathrm{C}-\mathrm{O}]$ is a 16 dimensional space. The identity follows by comparing coefficients.

To prove the identity for $F_{9}$, we begin by noting that

$$
f(-q)=q^{-1 / 24} \eta(z) .
$$

Using this fact together with (3.3), then, as above, we find that (1.5) is equivalent to

$$
\theta(z) F_{9}\left(q^{2}\right)=\theta(z)\left[\theta^{8}(9 z)-16 \frac{\eta^{16}(18 z)}{\eta^{8}(9 z)}+256 \frac{\eta^{16}(36 z)}{\eta^{8}(18 z)}+18 \frac{\eta^{12}(18 z)}{\eta^{4}(6 z)}\right] .
$$

To show that the right side, like the left, lies in $M_{9 / 2}\left(\tilde{\Gamma}_{0}(36)\right)$, it will suffice to show that the bracketed expression lies in $M_{8 / 2}\left(\tilde{\Gamma}_{0}(36)\right)=M_{4}\left(\Gamma_{0}(36)\right)$. For the first term, this follows by (3.2). For the three eta-quotients, this may verified using the aforementioned theorems of Gordon, Hughes, Newman and Ligozat. The dimension of $M_{9 / 2}\left(\tilde{\Gamma}_{0}(36)\right)$ is 26 , and the proof is completed by comparing coefficients.

\section{4. $F_{10}$}

The same techniques enable us to find an expression (albeit not as simple as those above) for $F_{10}(q)$.

Theorem 2. Let the notation be as in Section 1. Then

$$
\begin{aligned}
F_{10}(q) & =\frac{\varphi^{10}\left(\sqrt{q^{5}}\right)}{\varphi(\sqrt{q})}-8 \varphi^{5}\left(\sqrt{q^{5}}\right) \cdot q \frac{f^{5}\left(-q^{5}\right)}{f(-q)}-27 \varphi^{5}\left(\sqrt{q^{5}}\right) \cdot q^{2} \frac{f^{5}\left(-q^{10}\right)}{f\left(-q^{2}\right)} \\
& +125 \varphi\left(\sqrt{q^{5}}\right) \cdot q^{2} \frac{f^{5}\left(-q^{10}\right)}{f\left(-q^{2}\right)} \cdot \sqrt{q^{5}} \psi^{4}\left(q^{5}\right)-5 \varphi\left(\sqrt{q^{5}}\right) \cdot q^{2} \frac{f^{5}\left(-q^{10}\right)}{f\left(-q^{2}\right)} \cdot \sqrt{q} \psi^{4}(q) \\
& -17 \varphi(\sqrt{q}) \sqrt{q^{5}} \psi^{8}\left(\sqrt{q^{5}}\right)+2 \varphi(\sqrt{q}) \varphi^{4}\left(\sqrt{q^{5}}\right) \cdot \sqrt{q} \psi^{4}(q) \\
& -20 \varphi(\sqrt{q}) \cdot q^{4} \frac{f^{10}\left(-q^{10}\right)}{f^{2}\left(-q^{2}\right)}+5 \varphi(\sqrt{q}) \cdot q^{2} \frac{f^{10}\left(-q^{5}\right)}{f^{2}(-q)} .
\end{aligned}
$$


The proof proceeds as those above; we give only a brief sketch here. To exhibit the relevant identity between modular forms, we replace $q$ by $q^{2}$ in Theorem 2 , multiply both sides by $\theta(z)$, and use (3.3) and (3.5) to obtain

$$
\begin{aligned}
\theta(z) F_{10}\left(q^{2}\right) & =\theta^{10}(5 z)-8 \theta(z) \theta^{5}(5 z) \cdot \frac{\eta^{5}(10 z)}{\eta(2 z)}-27 \theta(z) \theta^{5}(5 z) \cdot \frac{\eta^{5}(20 z)}{\eta(4 z)} \\
& +125 \theta(z) \theta(5 z) \cdot \frac{\eta^{5}(20 z)}{\eta(4 z)} \cdot \frac{\eta^{8}(20 z)}{\eta^{4}(10 z)}-5 \theta(z) \theta(5 z) \cdot \frac{\eta^{5}(20 z)}{\eta(4 z)} \cdot \frac{\eta^{8}(4 z)}{\eta^{4}(2 z)} \\
& -17 \theta^{2}(z) \frac{\eta^{16}(10 z)}{\eta^{8}(5 z)}+2 \theta^{2}(z) \theta^{4}(5 z) \frac{\eta^{8}(4 z)}{\eta^{4}(2 z)}-20 \theta^{2}(z) \frac{\eta^{10}(20 z)}{\eta^{2}(4 z)} \\
& +5 \theta^{2}(z) \frac{\eta^{10}(10 z)}{\eta^{2}(2 z)} .
\end{aligned}
$$

To prove the last identity, we verify (using the methods of the last section) that each side of the proposed identity lies in $M_{5}\left(\Gamma_{0}(40), \chi_{-1}\right)$, and then compare a sufficient number of terms.

\section{REFERENCES}

[C-O] H. Cohen and J. Oesterlé, Dimensions des espaces de formes modulaires, Springer Lect. Notes 627 (1976), 69-78. MR 57:12396

[G-H] B. Gordon and K. Hughes, Multiplicative properties of eta-products II, Contemp. Math. 143 (1993), 415-430. MR 94a:11058

[K] N. Koblitz, Introduction to elliptic curves and modular forms, Springer Verlag, New York, 1984. MR 86c: 11040

[L] G. Ligozat, Courbes modulaires de genre 1, Bull. Soc. Math. France 43 (1972), 1-80. MR 54:5121

[O] K. Ono, On the circular summation of the eleventh powers of Ramanujan's theta function, J. Number Theory (to appear).

[Ram] S. Ramanujan, The lost notebook and other unpublished papers, Narosa Publ. House, New Dehli, 1988. MR 89j:01078

[Ran] S. Rangachari, On a result of Ramanujan on theta functions, J. Number Theory 48 (1994), 364-372. MR 95i:11038

[Sh] G. Shimura, On modular forms of half integral weight, Annals of Math. 97 (1973), 440-481. MR 48:10989

[S] S. Son, Circular summations of theta functions in Ramanujan's lost notebook, preprint.

Department of Mathematics, The Pennsylvania State University, University Park, PenNSYlVANia 16802-6401

E-mail address: ahlgren@math.psu.edu

Current address: Department of Mathematics, Colgate University, Hamilton, New York 13346 